\title{
A Comparative Study of Coagulation Systems in Newborn Animals
}

\author{
PATTI MASSICOTTE, LESLEY MITCHELL, AND MAUREEN ANDREW \\ Department of Pediatrics, McMaster University Medical Centre, Hamilton, Ontario, Canada
}

\begin{abstract}
Appropriate animal experimentation can enhance our understanding of thrombotic and hemorrhagic problems in the human neonate. Which newborn animal species' coagulation system most closely resembles the human neonate is unknown. The objective of the study was to assess the newborn coagulation system in four animal species and compare them with the human neonate. Blood samples were drawn on days 1 and 7 of life from lambs $(n=10)$, piglets, $(n=12)$, rabbit pups $(n=12)$, and beagle pups $(n=7)$. Coagulation screening tests, specific factor assays, and specific inhibitors of the coagulation system were measured. All factor assays were expressed as a percent of the respective species pooled adult plasma. The results from the animals were compared to normal values from our laboratory for healthy full-term infants. The coagulation systems of all species tested, except the rabbit pup, were immmature at birth with most factor levels being lower than the adult of their species. The coagulation systems were influenced by the postnatal age of the animal and the factor levels reached adult values in fewer days relative to the human. The coagulation system for the piglet most closely approximated the human neonate. The shared characteristics were prolonged screening tests, increased factor VIII:C, generally low levels for the contact and vitamin K-dependent factors, and low antithrombin III levels relative to the adult. The beagle pup also showed many similar characteristics but in contrast to the human neonate factor VIII:C and V were low on day 1 of life and prekallikrein was not measurable in the adult or newborn beagle. These data, especially the low antithrombin III levels, support the use of the piglet to investigate thrombotic problems in the neonate. (Pediatr Res 20: 961-965, 1986)
\end{abstract}

\section{Abbreviations}

APTT, activated partial thromboplastin time

PT, prothrombin time

TCT, thrombin clotting time

HMWK, high molecular weight kininogen

PK, prekallikrein

$\alpha_{2}$-AP, $\alpha_{2}$-antiplasmin

VIII:C, VIII procoagulant
AT-III, antithrombin III

The sick newborn infant is at risk of developing thrombotic and hemorrhagic problems in the postnatal period. The investigation of thrombotic and hemorrhagic problems in the neonate is often limited by the small size of the infant and the limited forms of investigation that are ethically acceptable in this group of patients. Despite the potential of animal models in investigating hemorrhagic and thrombotic problems in the neonate, there is limited information on the coagulation systems in newborn animals $(1,2)$. The objective of this study was to assess systematically the coagulation system in four newborn animal species and compare them with the human infant in order to determine which animal(s) would be most appropriate for investigation of hemorrhagic and thrombotic problems in the human neonate.

\section{MATERIALS AND METHODS}

Study population. Newborn lambs (mixed breed), piglets (mixed breed), rabbit pups (New Zealand White), and purebred beagle pups were studied on days 1 and 7 of life. An adult plasma pool was prepared for each species from 20 adult animals of both sexes. Blood was collected directly into $0.13 \mathrm{M}$ sodium citrate (1 part citrate to 9 parts blood except for the piglet for which the ratio was 1.35 citrate to 9 parts blood) from each animal by peripheral venipuncture or cardiac puncture. Reference values for healthy full-term infants from our laboratory were used for comparison (3).

Coagulation tests. The hematocrit was measured and platelet poor plasma prepared and stored at $-70^{\circ} \mathrm{C}$ in polypropylene tubes for batch assaying. Coagulation tests were measured using microtechniques which have been published in detail (4). These tests consisted of the PT, APTT, TCT, and specific coagulation factor activities, including factors II, V, VII, VIII:C, IX, X, XI, $\mathrm{XII}$, and HMW-K. The factor levels were assayed using onestage assays measuring the ability of the test plasma to correct human plasma known to be deficient in the factors to be tested. The fibrinogen concentration was measured as a clottable protein (5) and by the tryosine-release method (6). PK and the inhibitors, AT-III, and $\alpha_{2}$-AP were measured using established chromogenic assays (7-9).

The results of the coagulation tests were expressed as the mean \pm 1 SEM. Differences between day 1 and 7 values were analyzed by Student's unpaired or paired two-tailed $t$ test, as appropriate. For multiple comparisons between groups of animals the Bonferroni correction was used. $p$ values less than 0.05 were considered to be significant.

\section{RESULTS}

Received February 10, 1986; accepted May 20, 1986

Request for reprints Dr. M. Andrew, Department of Pediatrics, McMaster University Health Sciences Centre, Room HSC 3N27, 1200 Main Street West, Hamilton, Ontario L8N 3Z5, Canada.

This work was supported by Grant AN-4 from the Heart and Stroke Foundation of Ontario. M.A. is a scholar of the Canadian Heart Foundation and P.M. was supported by a Medical Research Council Summer Student Research Scholarship.
Newborn animals from each species were studied on days 1 and 7 of life. The same animals were studied on both days except for the rabbit pups whose small size necessitated cardiac puncture to obtain the blood samples. Data from our laboratory, for healthy full-term infants studied on days 1 and 5 of life were 
used for comparison in the figures and tables, with the day 5 infant values compared to the day 7 values in the animals. Between 45 and 60 human infants were studied at both time points for all assays.

Table 1 gives the number of newborn animals studied, their weights and hematocrits on day 1 of life. Only the piglet had a low hematocrit necessitating the adjustment of the amount of anticoagulant used. Table 2 compares the coagulation screening tests and specific factor assays from the pooled adult animal plasmas to the pooled adult human plasma. In general, the values compare well to previously published values (10). Some animal factor assays were nonlinear at higher concentrations when compared to the human but the assays became linear at more dilute concentrations. The reproducible values at the dilute concentrations were chosen as the true value. In the adult species, the fibrinogen concentration was quantitated by the tyrosine release method (Table 2). The newborn samples were measured as thrombin clottable protein and compared to their respective adult species standard curve. Fibrinogen levels for the piglet were lower than the other newborn animal species when measured as a clottable protein but gave a mean value of $1.28 \mathrm{~g} /$ liter when measured by the tyrosine release method. The lower values in the piglet may be related to the use of the bovine thrombin and/ or the presence of a fetal fibrinogen.

Figure 1 shows the coagulation screening test values in the four newborn animal species compared to the healthy full-term infant. The data have been expressed as a fraction of the adult value which is considered to be 1.0. In general, the PT and APTT are prolonged on day 1 compared to the respective adult species and these tests achieve adult or near adult values by day 7 of life

Table 1. Wt and hematocrits of newborn animals on day 1 of

\begin{tabular}{lrrc}
\multicolumn{4}{c}{ life } \\
\hline Species & \multicolumn{1}{c}{$n$} & \multicolumn{1}{c}{ Wt $(\mathrm{kg})^{*}$} & Hematocrit (\%)* \\
\hline Rabbit pup & 12 & $0.1 \pm 0.01$ & $41 \pm 0.8$ \\
Beagle pup & 7 & $0.3 \pm 0.04$ & $47 \pm 2.1$ \\
Lamb & 10 & $3.6 \pm 0.21$ & $35 \pm 1.8$ \\
Piglet & 12 & $1.28 \pm 0.06$ & $23 \pm 1.2$ \\
\hline
\end{tabular}

* Mean \pm SEM.

Table 2. Coagulation factor levels in four adult animal species compared with adult human

\begin{tabular}{lcccc}
\hline \multicolumn{1}{c}{ Factor } & Rabbit & Dog & Sheep & Pig \\
\hline Fibrinogen $(\mathrm{g} /$ liter $)$ & 2.86 & 3.39 & 3.00 & 3.70 \\
II $(\mu / \mathrm{ml})^{*}$ & 0.79 & $1.02 \dagger$ & 0.19 & 0.81 \\
V $(\mu / \mathrm{ml})^{*}$ & 4.79 & 3.04 & $1.81 \dagger$ & 1.98 \\
VII $(\mu / \mathrm{ml})^{*}$ & $1.43 \dagger$ & 4.75 & 0.13 & 1.39 \\
VIII:C $(\mu / \mathrm{ml})^{*}$ & $1.70 \dagger$ & 6.19 & 7.90 & 3.78 \\
IX $(\mu / \mathrm{ml})^{*}$ & $1.00 \dagger$ & 1.00 & $1.19 \dagger$ & $1.70 \dagger$ \\
X $(\mu / \mathrm{ml})^{*}$ & $0.77 \dagger$ & $1.14 \dagger$ & $0.50 \dagger$ & $1.14 \dagger$ \\
XI $(\mu / \mathrm{ml})^{*}$ & 2.98 & 3.32 & 0.82 & 1.50 \\
XII $(\mu / \mathrm{ml})^{*}$ & $0.31 \dagger$ & $1.34 \dagger$ & 2.53 & $2.24 \dagger$ \\
PK $(\mu / \mathrm{ml})^{*}$ & $<0.10$ & $<0.10$ & 0.16 & 1.02 \\
HMWK $(\mu / \mathrm{ml})^{*}$ & $<0.10$ & $1.0 \dagger$ & 0.85 & $1.67 \dagger$ \\
AT-III $(\mu / \mathrm{ml})^{*}$ & 0.79 & 0.82 & 0.63 & 0.76 \\
$\alpha_{2}$-AP $(\mu / \mathrm{ml})^{*}$ & 1.06 & 1.01 & $1.25 \dagger$ & 0.72 \\
PT $(\mathrm{s})$ & 9.0 & 7.0 & 13.0 & 13.0 \\
PTT $(\mathrm{s})$ & 31.0 & 18.0 & 29.0 & 21.0 \\
TCT $(\mathrm{s})$ & 18.0 & 16.0 & 17.0 & 17.0 \\
\hline
\end{tabular}

* The factor levels in human pooled plasma were defined as $1 \mathrm{U} / \mathrm{ml}$ and the pooled plasma from each animal species was compared to the human.

$\dagger$ These factors were nonlinear at low dilutions (i.e. 1/10) when compared to the human pool. At higher dilutions (i.e. 1/80,1/100) the factor assay results were replicable. The results were calculated from the higher dilutions.

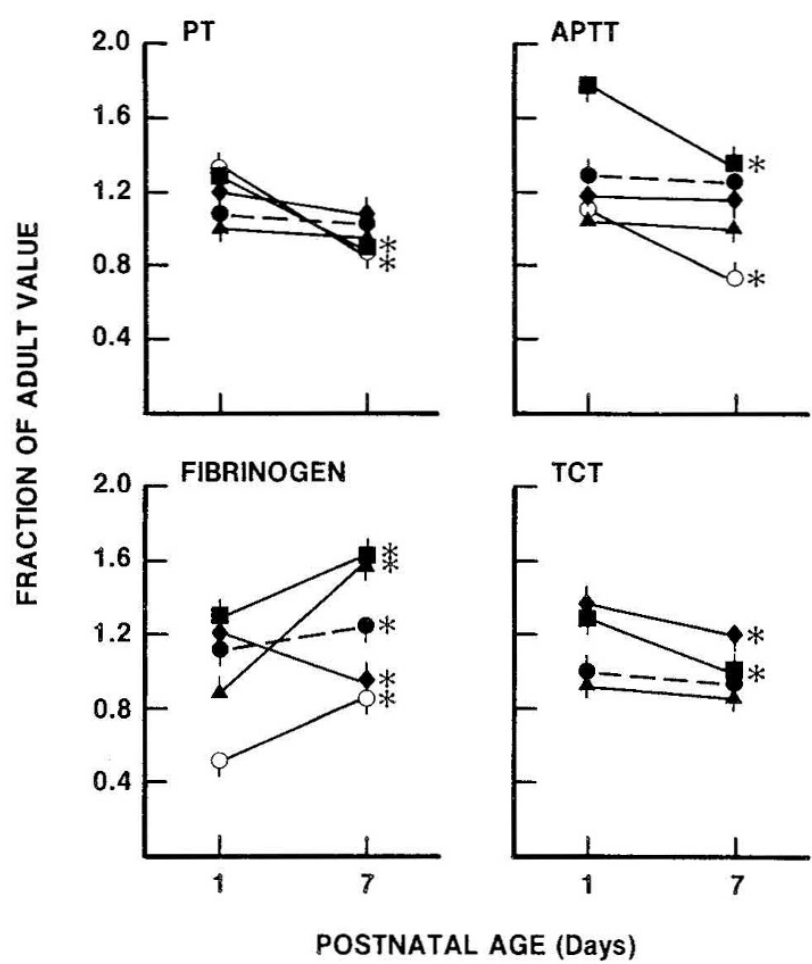

Fig. 1. The APTT, PT, fibrinogen level, and TCT in four newborn animal species and healthy full-term infants in the postnatal period. The values are expressed as a fraction of the adult species value which is considered to be 1.0. The day 1 values were compared to the day 7 values for the rabbit pup $\downarrow$, piglet $O$, lamb $\boldsymbol{\Lambda}$, beagle pup $\boldsymbol{\square}$, and human infant $\bullet$. The data are expressed as mean values + SEM with a $p$ value $<0.05$ indicated $(*)$.

for the animals. For the human infant, the PT shortens to adult values by day 7 , however, the APTT is still prolonged on day 7 compared to the adult. The fibrinogen levels are in the adult range for all species except the piglet on day 1 and the fibrinogen levels continue to rise by day 7 of life. The TCTs are slightly prolonged in the newborn animal species on day 1 , but reach adult values by day 7 . The human infant has an adult value for the TCT on day 1 of life. The TCT could not be accurately measured in the piglet due to the relative insensitivity of the piglet fibrinogen to bovine thrombin.

Figure 2 shows the vitamin K-dependent factor values in the four newborn animal species compared to the healthy full-term infant. Adult values for each species are considered to be 1.0 $\mathrm{U} / \mathrm{ml}$. None of the animal species received vitamin $\mathrm{K}$ at birth, whereas all of the human infants did. The day 1 values for factor II are unusual in that the day 1 values are above the adult values for the rabbit pup, piglet, and lamb. The day 1 values for factors VII and $\mathrm{X}$ in the rabbit pup are also above the adult range whereas the other animal species are similar to the human infant in that the vitamin $\mathrm{K}$-dependent factor values are below $60 \%$ of adult values. In general, all species and the human infant show a rise in the vitamin K-depenent factor levels by day 7 .

Figure 3 shows the contact factor values in the four newborn animal species compared to the human full-term infant. On day 1 of life, the levels for factors XII, XI, and HMW-K in the piglet, lamb, and beagle pup are lower than adult values and these factor levels are increasing by day 7 of life. The rabbit pup has high levels for factors XI and XII and unmeasurable values for PK and HMW-K (11) (Table 2). We also found that PK is not present in the adult dog (Table 2).

Figure 4 shows the levels for the cofactors, factor VIII:C and factor V, and for the inhibitors, AT-III and $\alpha_{2}$-AP in the four animal species compared to the healthy full-term infant. A wide 


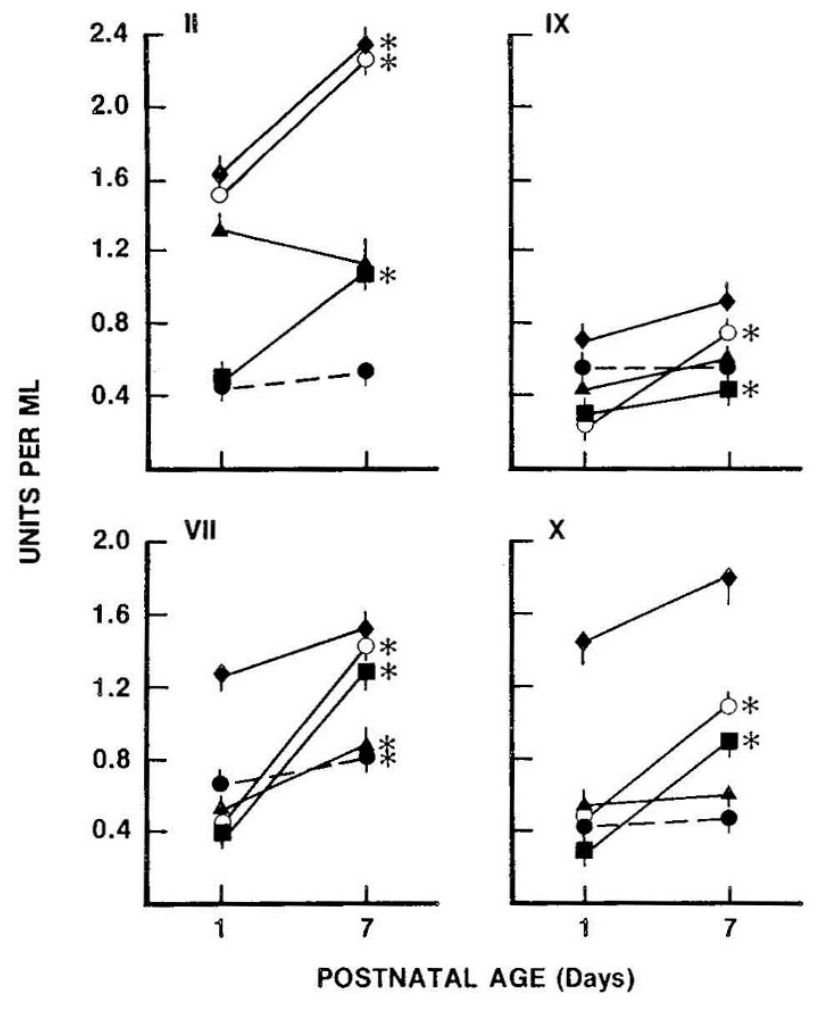

Fig. 2. The vitamin K-dependent factors (II, VII, IX, X) in four newborn animal species and the healthy fullterm infant in the postnatal period. The values are expressed as a fraction of the adult species value which is considered to be $1.0 \mathrm{U} / \mathrm{ml}$. The day 1 values were compared to the day 7 values for the rabbit pups $\downarrow$, piglet $O$, lamb $\boldsymbol{\Lambda}$, beagle puppy $\boldsymbol{\square}$, and human infant $\bullet$. The data are expressed as mean values \pm SEM with a $p$ value $<0.05$ indicated $(*)$.

range of levels for these factors is seen between the species. The piglet has values for factor VIII:C and factor V which most closely approximate the newborn infant on day 1 of life. In addition, both the piglet and beagle puppy have low levels of AT-III on day 1 of life whereas the rabbit and the sheep already have adult levels of these proteins.

The four newborn animal species were further compared to the human infant on day 1 of life. The values for each of the factor and inhibitor levels for each species were meaned to give an average ratio for a specific species (Fig. 5). The piglet and beagle puppy had overall ratios that closely approximated the infant whereas the rabbit pup and lamb were significantly different.

\section{DISCUSSION}

The study of fetal and neonatal coagulation using animal models is an important extension of research focused on hemorrhagic and thrombotic problems in the newborn. Although the coagulation system of the fetal lamb in utero has been well described (1), there is very little information on the coagulation systems of newborn animals in the immediate postnatal period (2). This study has examined the coagulation system in four newborn animal species in depth and compared them to the adult of their species and to the human neonate. The four species studied were chosen as they have been used to investigate specific hemorrhagic or thrombotic problems in the neonate. Our findings show that the piglet and beagle puppy have coagulation systems which most closely approximate the human infant on day 1 of life (Fig. 5).

The most extensively studied model of fetal and neonatal hemostasis is the fetal lamb $(1,12)$. This model has been used to investigate potential hemostatic complications in the fetus, such as maternal use of corticosteroids (13), maternal hypoxia (14, 15), intraventricular hemorrhage (16), and thrombosis (17). While this model is very useful for investigating disorders in the fetus, it may have certain limitations when used to investigate the postnatal coagulation system in the newborn. Our study shows that as early as day 1 of life the coagulation system in the newborn lamb is significantly different from the human infant (Fig. 5) and the levels for AT-III are in the adult range. The latter is in marked contrast to the human neonate and could potentially limit the usefulness of the newborn lamb for investigating thrombotic problems in the newborn.

The newborn beagle puppy has been used as a model to investigate intraventricular hemorrhage $(18-20)$. There is no information regarding the coagulation system in the beagle puppy, although the coagulation systems in other newborn dog species have been partially investigated $(2,21)$. This information is important because there is an association between coagulation abnormalities and intraventriuclar hemorrhage (22-25) and the beagle puppy may provide a useful model for the investigation of this association. Our study shows that the beagle puppy is very similar to the human neonate for most coagulation factors including low levels of AT-III. The limitation of this model is that there is no measurable PK and factors VIII:C and V are approximately $30 \%$ lower than the human neonate on day 1 of life. These dissimilarities between the beagle puppy and the human infant may be important depending on which pathologic question is being addressed.

The adult rabbit has been used extensively as a model for human adult thrombotic and hemorrhagic diseases and the rabbit pup has been used as a model for prematurity (26). The studies reported herein expand on the previous information on the rabbit pup coagulation system $(2,27)$ and suggest that the rabbit pup

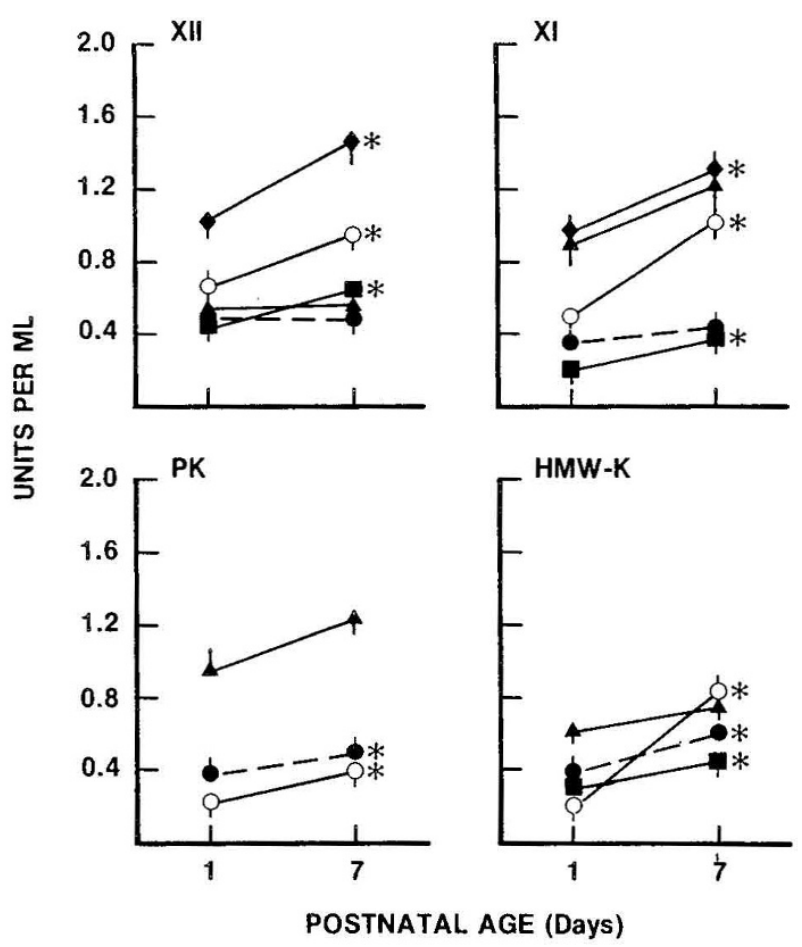

Fig. 3. The contact factors (XII, XI, PK, HMW-K) in four newborn animal species and healthy full-term infant in the postnatal period. The values are expressed as a fraction of the adult species value which is considered to be $1.0 \mathrm{U} / \mathrm{ml}$. The day 1 values were compared to the day 7 values for the rabbit pup $\downarrow$, piglet $O$, lamb $\boldsymbol{\Delta}$, beagle puppy $\mathbf{\square}$, and human infant $\bullet$. The data are expressed as mean values \pm SEM with a $p$ value $<0.05$ indicated $\left(^{*}\right)$. 


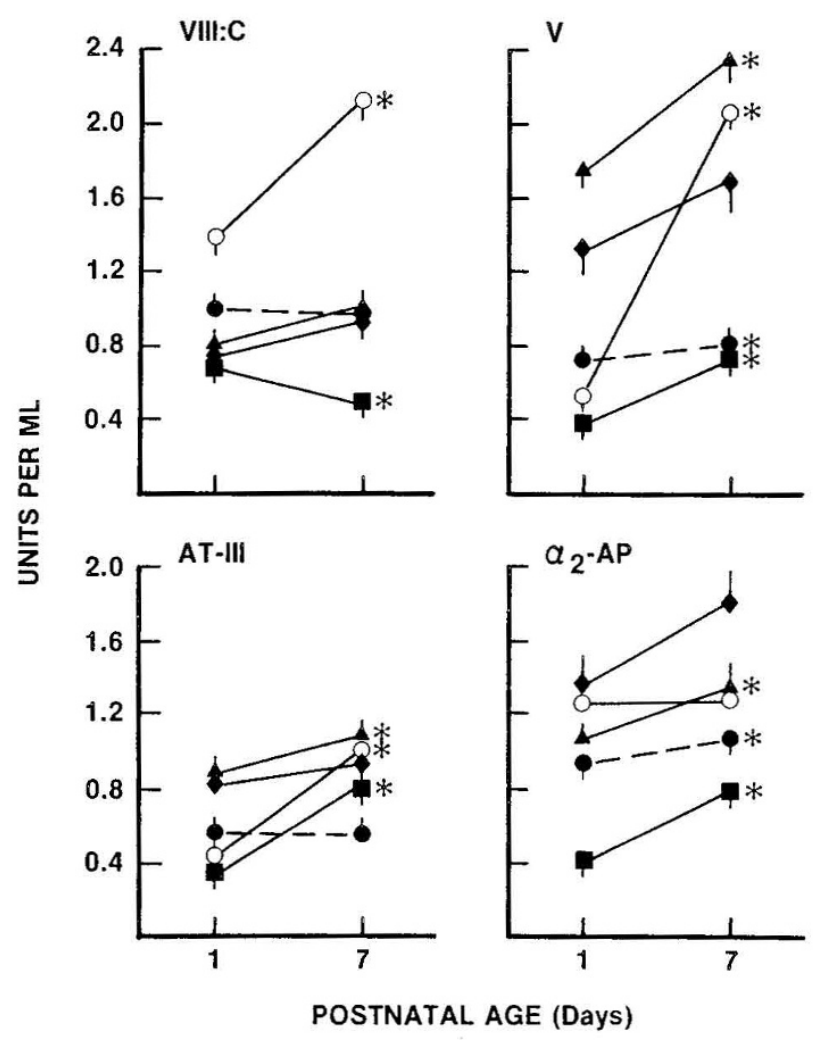

Fig. 4. The factor VIII:C and V and the inhibitors AT-III and $\alpha_{2}$-AP in four newborn animal species and healthy full-term infants in the postnatal period. The values are expressed as a fraction of the adult species value which is considered to be $1.0 \mathrm{U} / \mathrm{ml}$. The day 1 values are compared to the day 7 values for the rabbit pup $\downarrow$, piglet $\mathrm{O}$, lamb $\boldsymbol{\Lambda}$, beagle puppy and human infant $\boldsymbol{\bullet}$. The data are expressed as mean values \pm SEM with a $p$ value $<0.05$ indicated $\left({ }^{*}\right)$.

has limited value as a model of neonatal coagulation. The rabbit pup has near or above adult levels for most coagulation factors at birth and the balance between specific factors and inhibitors is not the same as in the human neonate.

The hemostatic system in the pig is very similar to that of the human adult (28). The physiologic similarities between the adult human and adult porcine hemostatic systems, the appropriate size of the newborn piglet, and the close similarity between the piglet and human infant for the coagulation system suggest that the newborn piglet may be a suitable model to use to investigate specific hemorrhagic and thrombotic problems in the newborn. In particular, the low AT-III levels and the presence of a possible "fetal" fibrinogen in the piglet may be of particular importance when choosing a newborn thrombosis model (29). The rapid maturation of the piglet coagulation system suggests that this model may only be appropriate for a brief time in the immediate postnatal period.

In summary, the coagulation systems of the beagle puppy, piglet, and lamb are relatively immature on day 1 of life compared to the respective adult species. The postnatal development to adult values occurs more rapidly in all animal species when compared to the human infant. This rapid change likely limits their usefulness to the immediate postnatal period. The coagulation systems in the beagle puppy and piglet most closely approximate the human infant whereas the newborn rabbit had many important differences (Fig. 5). The results of this study expand on the data base available from which one can choose an appropriate animal model to investigate hemorrhagic or thrombotic complications in the neonate.

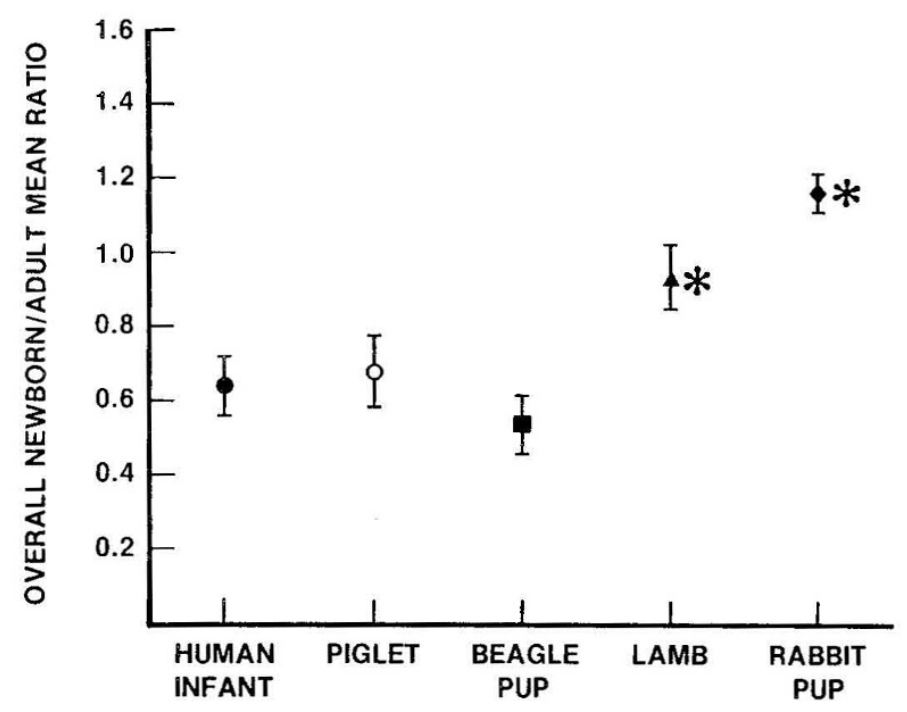

Fig. 5. An overall comparison of the coagulation systems in the four animal species and the human infant on day 1 of life. The newborn to adult ratio was calculated for each species by meaning the values for each of the factor and inhibitor levels. Using the piglet as an example, the day 1 values in $\mathrm{U} / \mathrm{ml}$ for fibrinogen, factors II, V, VII, VIII, IX, X, $\mathrm{XI}, \mathrm{XII}, \mathrm{PK}, \mathrm{HMW}-\mathrm{K}$ AT-III, and $\alpha_{2}$-AP were meaned to give an overall newborn to adult pig mean ratio of 0.67 . The mean values for each animal species were compared to the human infant using an unpaired Student's $t$ test with a Bonferroni correction. The data are expressed as mean values \pm SEM with a $p$ value of $<0.05$ indicated $(*)$.

Acknowledgments. The authors acknowledge the secretarial assistance of Mrs. B. Lahie and Mrs. J. Butera, and the technical assistance of Miss M. Smith.

\section{REFERENCES}

1. Kisker CT, Robillard JE, Clarke WR 1981 Development of blood coagulation - a fetal lamb model. Pediatr Res 15:1045-1050

2. Hathaway WE, Hathaway HS, Belbusen LP 1964 Coagulation factors in newborn animals. J Lab Clin Med 63:784-790

3. Andrew M, Paes B, Johnston M, Milner R, Powers P, Toliefson D 1985 The normal development of the coagulation system in fullterm infants. Thromb Haemost 54:259(abstr)

4. Johnston M, Zipursky A 1980 Microtechnology for the study of the blood coagulation system in newborn infants. Can Med Tech 42:159-164

5. Clauss A 1957 Gerinnungsphysiologische Shnellmethode zur best immung des Fibrinogens. Acta Haematol (Basel) 17:237-246

6. Astrup T, Brakman P, Nissen J 1965 The estimation of fibrinogen-a revision. Scand J Clin Lab Invest 17:57-65

7. Kluft C 1978 Determination of prekallikrein in human plasma: optimal conditions for activating prekallikrein. J Lab Clin Med 91:83-93

8. Abildgaard U, Lie M, Odegard OR 1977 Antithrombin (heparin cofactor) assay with new chromogenic substrates (S-2238 and chromozym-Th). Thromb Res 11:549-553

9. Tiger-Nilsson AC, Fiberger P, Gyzander E 1977 Determination of a new rapid plasmin inhibitor in human blood by means of a plasmin specific tripeptide substrate. Scand J Clin Invest 37:403-409

10. Dobbs WJ 1981 Second international registry of animal models of thrombosis and hemorrhagic diseases. ILAR News XXIV:R1-R50

11. Saito H, Goldsmith G, Ratnoff O 1974 Fletcher factor activity in plasmas of various species (38378). Proc Soc Exp Biol Med 147:519-523

12. Fantel P, Ward HA 1960 Clotting activity of maternal and fetal sheep blood. J Physiol 150:607-620

13. Kisker CT, Robillard JE 1983 Glucocorticoid stimulation of blood coagulation factor activities in the fetal lamb. J Lab Clin Med 101:569-575

14. Kisker CT, Robillard JE, Clarke WR 1982 Blood coagulation changes following hypoxemia in the near term fetal lamb. Pediatr Res 16:732-739

15. Kisker CT, Robillard JE, Clarke WR 1982 Blood coagulation changes after hypoxemia: a fetal lamb model. Pediatr Res 16:8-12

16. Wheeler AS, Sadri S, Gutsche BB, Devore JS, David-Mian Z, Latyshevsky H 1979 Intracranial hemorrhage following intravenous administration of sodium bicarbonate or saline solution in the newborn lamb asphyxiated in utero. Anaesthesiology 51:517-521

17. Hecker JF, Fisk GC, Gupta JM, Abrahams N, Kokkington RA, Lewis BR 
1979 Thrombus formation on catheters in newborn lambs. Anaesth Intensive Care 7:239-243

18. Ment LR, Stewart WB, Duncan CC, Scott DT, Lambrecht R 1984 Beagle puppy model of intraventricular hemorrhage. Effects of indomethacin on local cerebral glucose utilization. J Neurosurg 60:737-743

19. Goddard J, Lewis RM, Alcala H, Zeller RS 1980 Intraventricular hemorrhage-an animal model. Biol Neonate 37:39-52

20. Ment LR, Stewart WB, Duncan CC, Lambrecht R 1982 Beagle puppy model of intraventricular hemorrhage. J Neurosurg 57:219-223

21. Hathaway WE, Henderson BJ 1968 Effects of hypoxia on coagulation factors in the newborn dog. Biol Neonate 13:26-33

22. McDonald MM, Johnston ML, Rumack CM, Koops BL, VanGuggenheim MA, Babb C, Hathaway WE 1984 Role of coagulopathy in newborn intracranial hemorrhage. Pediatrics 74:26-31
23. Setzer ES, Webb IB, Bassencar JW, Reeder JD, Mehta PS, Eitzman DV 1982 Platelet dysfunction and coagulopathy in intraventricular hemorrhage in the premature infant. J Pediatr 100:599-605

24. Beverley DW, Chance GW, Inwood MJ, Schaus M, O'Keefe B 1984 Intraventricular hemorrhage and haemostatic defects. Arch Dis Child 59:444-448

25. Castle V, Andrew M, Kelton J, Carter C, Giron D 1984 A prospective study of thrombocytopenia in the neonate. Blood 64:853(abstr)

26. Lorenzo $\mathrm{AB} 1985$ The preterm rabbit: a model for the study of acute and chronic effects of premature birth. Pediatr Res 19:201-205

27. Chaignier E, Baguet J, Dessapt B, Bellevile J, Descotes Y, Eloy R 1981 Skin healing and fibrin stabilizing factor blood levels in the rabbit fetus. J Surg Res 31:415-432

28. Dodds JW 1982 The pig model for biomedical research. Fed Proc 41:247-256

29. Barr RD 1978 Fetal fibrinogen: fact or fancy? Biomedicine $28: 245-248$ 